\title{
What determines galactic evolution?
}

\author{
Francesca Matteucci \\ Department of Astronomy, University of Trieste \\ Via G.B. Tiepolo 11, 34131 Trieste, Italy
}

\begin{abstract}
We are briefly introducing the most important ingredients to study galactic evolution. In particular the roles of star formation, nucleosynthesis and gas flows. Then we are discussing the two different approaches to galactic evolution: the stellar population approach (chemical evolution models) and the hierarchical clustering scenario for galaxy formation. It is shown that there are still some controversial points in the two approaches, as evident in the brief summary of the discussion.
\end{abstract}

\section{Introduction}

Many parameters influence galactic evolution, in particular, we remind here some of the most important ones:

- Initial conditions: closed or open model, initial chemical composition and gas temperature.

- Gravity (baryonic and dark matter).

- Star formation history (star formation rate plus initial mass function).

- Stellar nucleosynthesis (roles of SNe).

- ISM processes (heating, cooling, mixing, SN feedback).

- Gas dynamics (enrichment of the IGM, ICM).

We intend to discuss briefly some of these items in connection with the constraints that they can impose on galaxy formation models. In particular, we would like to compare the predictions of current chemical evolution models and those of models based on the hierarchical clustering (HC) scenario with some relevant observational constraints relative to stellar populations in ellipticals and spirals.

(C) 2018 Kluwer Academic Publishers. Printed in the Netherlands. 
Matteucci

\section{Stellar nucleosynthesis}

Stellar nucleosynthesis is a fundamental parameter in driving galactic chemical evolution by means of the stellar yields. Particularly important is to study the role of stars with different lifetimes in enriching the interstellar medium (ISM). In particular, the different roles played by $\mathrm{SNe}$ of different type allow us, together with the star formation history $(\mathrm{SFH})$, to interpret the abundance patterns in galaxies. What we need to know is what elements are produced by stars of different masses and on which timescales:

- Low and intermediate mass stars $\left(0.8 \leq M / M_{\odot} \leq 8\right)$ are responsible for the production of ${ }^{4} \mathrm{He}, \mathrm{C}, \mathrm{N}$ and heavy s-process elements $(A>90)$. The lifetimes of these stars range from a Hubble time to $\sim 3 \cdot 10^{7}$ years.

- Type II SNe $\left(M \geq 10 M_{\odot}\right)$ produce mainly $\alpha$-elements $(\mathrm{O}, \mathrm{Ne}$, $\mathrm{Mg}, \mathrm{Si}, \mathrm{S}, \mathrm{Ca})$, part of Fe, light s-process $(\mathrm{A}<90)$ and r-process elements. Their lifetimes are less than $\sim 3 \cdot 10^{7}$ years.

- Type Ia SNe, which are believed to originate from C-deflagration in $\mathrm{C}-\mathrm{O}$ white dwarfs, produce mainly Fe-peak elements $(\sim 0.6-$ $0.7 M_{\odot}$ of $\mathrm{Fe}$ ). These $\mathrm{SNe}$ restore their products with a time delay relative to Type II SNe which can vary from $3 \cdot 10^{7}$ to 15 Gyr. Type Ia $\mathrm{SNe}$ are probably responsible for producing $\mathrm{Fe}$ in the universe unless the IMF is strongly biased towards massive stars. Their lifetimes are the same as those of low and intermediate mass stars.

Many detailed predictions about element production in stars of all masses are now available (see Matteucci 2001 for a review on this topic).

\section{The star formation history}

The SFH in a galaxy determines the evolution of the ISM and its chemical composition. The abundance patterns measured in galaxies (e.g. abundance ratios versus absolute abundances) are strongly influenced by their SFH. In fact, the time-delay between Type Ia and II SNe in restoring their nucleosynthesis products, together with the SFH determine completely the $[\alpha / \mathrm{Fe}]$ versus $[\mathrm{Fe} / \mathrm{H}]$ distributions or any other similar abundance pattern. The influence of the SFH occurs via the absolute abundances whereas the abundance ratios are independent of the SFH but depend upon the assumed stellar lifetimes, initial mass function (IMF) and stellar nucleosynthesis. The combination of stellar 


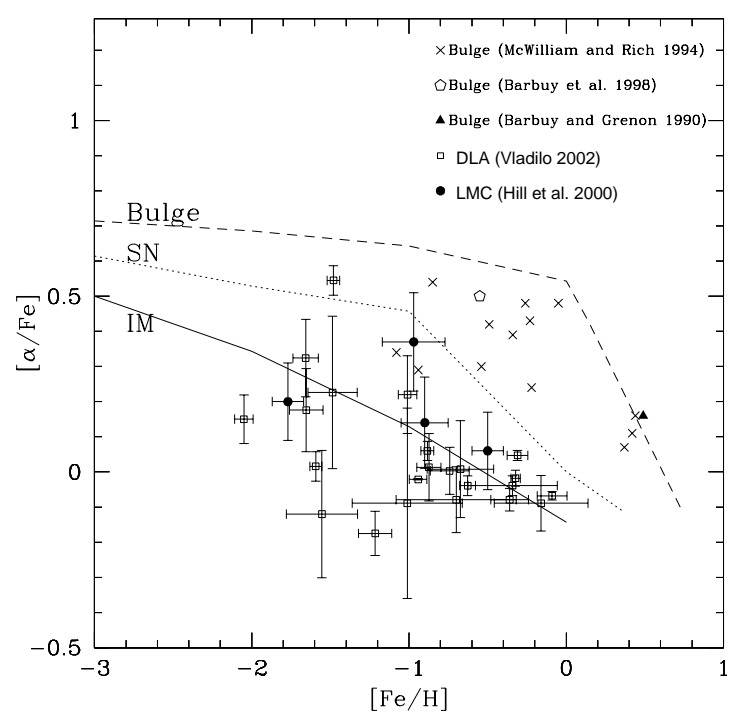

Figure 1. The predicted $[\alpha / \mathrm{Fe}]$ ratios for three different histories of star formation but equal IMF and nucleosynthesis.

nucleosynthesis and IMF represents the yields per stellar generation. The SFH influences the abundance ratios vs. abundances diagrams in the following way: galaxies with low star formation either in bursts or continuous (spirals and irregulars) will show a short plateau in $[\alpha / \mathrm{Fe}]$ ratio at very low metallicities, whereas galaxies with strong and fast SF will have a longer plateau for $[\alpha / \mathrm{Fe}]$ (bulges, ellipticals). This fact can be used to infer the nature of high redshift objects, for example just by comparing the predicted tracks for galaxies of different morphological type with the observed abundances in Damped Lyman- $\alpha$ objects (DLAs). In figure 1 we show the predictions of the $[\alpha / \mathrm{Fe}]$ ratio as a function of $[\mathrm{Fe} / \mathrm{H}]$ for different SFHs. In particular, for a spheroid (bulge or elliptical), the solar vicinity and a Magellanic irregular galaxy. For comparison are shown data points for stars and clusters in the bulge, data for the LMC and DLAs. The data seem to be in agreement with these predictions. Interestingly, the data points for the DLAs seem to fall preferentially on the curve relative to irregular galaxies, thus suggesting a possible identification of DLAs with such galaxies (see also Matteucci et al. 1997; Calura et al. 2002). 


\section{The IMF}

The IMF is a very crucial parameter in galactic evolution, and one should never try to change it unless it is absolutely necessary. For example, a time variable IMF could explain the curves of Fig. 1 without invoking any time delay effect between SNeII and Ia in galactic enrichment. However, this would imply a quite "ad hoc" variation of the IMF. One open problem is to assess if the IMF is universal. In particular, it is not clear yet whether there are differences in the IMF inside galaxies and/or among galaxies. In our opinion there are probably IMF variations among galaxies, whereas we cannot claim to have evidence for a variable (space and/or time) IMF in the Milky Way (see Chiappini et al. 2000 for arguments against the variation of the IMF).

\section{SN feedback}

How much energy is transferred from $\mathrm{SNe}$ and stellar winds into the ISM? We would like to know the answer to this question, since it is crucial for understanding galactic evolution. In fact, the energy deposited by stellar winds and SNe into the ISM may induce gas outflows in galaxies, which in turn are likely to stop the star formation for prolonged periods. Unfortunately, the efficiency of energy transfer is largely unknown and always parametrized in all the models of galactic evolution.

\section{Gas dynamics}

Gas dynamics is crucial in determining the evolution of galaxies since it studies the fate of the ISM. Gas infall and outflow are very important parameters: i) infall of extragalactic material is required to explain the G-dwarf metallicity distribution in the solar vicinity, ii) biased

infall is required to explain the formation of the Galactic disk and of disks in general (Chiappini et al. 1997; Boissier \& Prantzos 1998), iii) galactic winds are likely to occurr in ellipticals and in dwarf starburst galaxies and enrich the IGM/ICM in metals The outflows can affect the $[\alpha / \mathrm{Fe}]$ ratios inside and outside galaxies if the outflows are differentially enriched in heavy elements (Recchi et al. 2000). 


\section{Monolithic vs. Hierarchical}

The modern version of the so-called monolithic model for the formation of elliptical galaxies is not necessarily assuming that the galaxies form by the collapse of a single gas cloud but rather that ellipticals form at high redshift and on shorter timescales relative to spirals, and that they are assembled out of gas and not of preexisting stars. In particular, an early monolithic collapse of a gas cloud or early merging of lumps of gas, where dissipation plays a fundamental role are equivalent (Larson 1974; Arimoto \& Yoshii 1987; Matteucci \& Tornambè 1987). In the classical scenario for the formation of ellipticals, the star formation is assumed to stop after a galactic wind develops and all the residual gas is lost, and the galaxies are evolving passively since then.

The timescales required to develop a wind from constraints on the properties of the stellar populations in local ellipticals are less than or equal to 1 Gyr (Pipino et al. 2002). Further star formation is not excluded after the wind, but it should not be relevant to the evolution of the stellar populations. The winds are triggered by the energy injected primarily by supernova explosion and secondarily by stellar winds. Clearly, the prescriptions for the SN feedback are a crucial ingredients in these models and are fixed by reproducing the majority of the properties of the stellar populations in ellipticals as well as the chemical enrichment in the ICM. The appealing aspect of such models for the formation of ellipticals is that they can explain the majority of the observational constraints relative to stellar populations: colormagnitude relations, Fundamental Plane and the increase of the $[\alpha / \mathrm{Fe}]$ ratio with galactic mass (see also the detailed discussion by Peebles 2002 and references therein).

On the other hand, hierarchical models of galaxy formation have the advantage of operating in a cosmological context. Simulations of hierarchical galaxy formation in a cold dark matter (CDM) universe are based on semi-analytical models operating in the framework of the Press-Schechter theory. These models predict that galaxies form on a wide redshift range and that massive ones form preferentially at late times (e.g. Kauffmann et al. 1993). In particular, elliptical galaxies should form by merger of early formed stellar systems such as spiral galaxies, although a burst of star formation can occur during the major merging, where $\sim 30 \%$ of the stars can be formed (Kauffmann 1996).

It is clear that at least qualitatively the two approaches seem to predict the opposite trends: the hierarchical scheme predicts that spirals form before ellipticals which continue to assemble until recent times, as opposed to the monolithic model approach which predicts that ellipticals form on shorter timescales than spirals. 


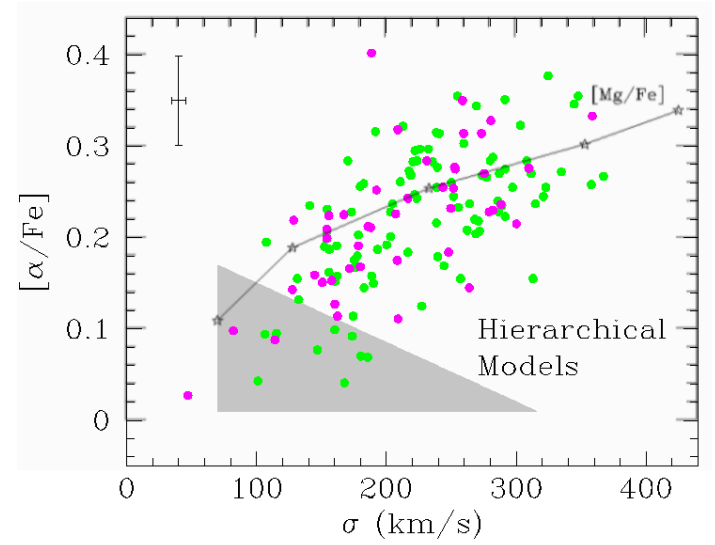

Figure 2. Predicted and observed $[\alpha / \mathrm{Fe}]$ vs. $\sigma$ relations. The line represents the prediction of the so-called monolithic model whereas the shaded area represents the predictions of a model assuming that ellipticals formed by merging. The figure is adapted from Thomas et al. (2002).

In figure 2 we show the predictions by a model of chemical evolution for ellipticals (Pipino \& Matteucci this conference) compared with the prediction by a model assuming the SFH derived from semi-analytical models of galaxy formation (Thomas 1999; Thomas et al. 2002) and with the observational data. As it is clearly shown in the figure, hierarchical models predict the opposite of what is observed, whereas the chemical evolution models well reproduce the $[\alpha / \mathrm{Fe}]$ versus central velocity dispersion $(\sigma)$ relation. In particular, the $[\alpha / \mathrm{Fe}]$ trend can be obtained under the assumption that more massive ellipticals are older than small ellipticals, in other words that massive ellipticals form on shorter timescales (Matteucci 1994). In this case, in fact, Type Ia SNe in massive ellipticals do not have time to substantially pollute the ISM before the occurrence of the galactic wind. The high observed value of the $[\alpha / \mathrm{Fe}]$ ratio in massive ellipticals requires that these objects formed on timescales of only few $10^{8}$ years.

Disk galaxies, on the other hand, should form on much longer timescales as indicated by successfull models of chemical evolution of the Galaxy, which suggest timescales as long as 7-8 Gyr for the local disk (Chiappini et al. 2001). Recent work by Boissier et al. (2001) has indicated that spiral galaxies in general seem to show the same tendency of ellipticals, in the sense that massive spirals are older than smaller ones. This conclusion is forced by several observational facts: in particular, larger disks are redder than smaller ones, have less gas and were more active in the past in producing stars than lower mass disks, and the efficiency of star formation seems to be constant among disk galaxies. 
The main difference between hierarchical and "monolithic" approach seems therefore to reside in the timescales for galaxy formation and the redshift of formation of different objects. ¿From the observational point of view there are several facts which indicate that ellipticals formed at early times and on a short timescale, some of these are (for a more extensive discussion see Peebles, 2002): i) the tightness of the colorcentral velocity dispersion relation found for Virgo and Coma galaxies (Bower et al. 1992) indicate that ellipticals in clusters have formed on timescales not longer than 2 Gyr. The same seems to hold for field ellipticals, altough the timescales for these latter should be 1 Gyr older (Bernardi et al. 1998). ii) The thinness of the fundamental plane for ellipticals in the same two clusters (Renzini and Ciotti 1993) also argues in favor of a short period of formation for ellipticals. However, it has been suggested that the tight relations in the fundamental plane could be due to a conspiracy of age and metallicity, in the sense that there is an age-metallicity anticorrelation implying that more metal rich galaxies are younger (Trager et al. 1998; Ferreras et al. 1999; Trager et al. 2000). iii) The thightness of the color-magnitude relation for ellipticals in clusters up to $z \sim 1$ (Kodama et al. 1998; Stanford et al. 1998), iv) as well as the modest passive evolution measured for cluster ellipticals at intermediate redshift (van Dokkum \& Franx 1996; Bender et al. 1996), argue in favor of an early formation of ellipticals. v) The Lyman-break and SCUBA galaxies found at high redshift, where the $\mathrm{SFR} \sim 40-1000 M_{\odot} y r^{-1}$ (Steidel et al. 1999; Pettini et al. 2002: Blain et al. 1999) can be the young ellipticals (Matteucci and Pipino, 2002).

However, there are also arguments in favor of a formation of ellipticals at low redshift although they seem weaker than those for the formation at high redshift. They are: a) the relative large values of the $H_{\beta}$ index measured in a sample of nearby ellipticals which could indicate prolonged star formation activity up to 2 Gyr ago (Gonzalez 1993; Faber et al. 1995; Trager et al. 1998) but see Maraston and Thomas (2000) for an alternative explanation.

b) The apparent paucity of high luminosity ellipticals at $z \sim 1$ compared to now (Kauffmann et al. 1996; Zepf, 1997; Menanteau et al. 1998; Franceschini et al. 1998). However, Daddi et al. (2000) showed that the observed surface density of extremely red objects (EROs) indicates that the bulk of these objects are passively evolving ellipticals, implying that that most field ellipticals were fully assembled already by $z \sim 2.5$. 


\section{Discussion}

In conclusion, we list some of the most relevant still open questions in galaxy evolution with the intent of stimulating a discussion. The questions are: Is the SFR depending on the galactic mass as suggested by observational evidences? Is the IMF universal? Are Population III stars necessary to explain the abundance patterns in galaxies? Are galactic winds from dwarf galaxies really important for the IGM/ICM enrichment? What are the DLAs, the Lyman-break and SCUBA galaxies? Is inhomogeneous mixing important in the early galactic evolution? Can the hierarchical scheme for galaxy formation reproduce real galaxies?

At this point the discussion started and was concentrated on the last of the open questions listed above, in particular on the comparison between hierarchical and monolithic models for galaxy formation.

Daniel Thomas started the discussion by showing a summary of observations which indicate some problems connected with hierarchical clustering models for the formation of galaxies. Some of them were addressed as "classical arguments", whereas others were addressed as "new arguments". The classical arguments are: missing satellites, angular momentum, cuspy cores. The new arguments are: the color-magnitude relation, the Fundamental Plane, $[\alpha / \mathrm{Fe}]$-sigma relation, anti-hierarchical ages of ellipticals, ages of bulges, chemical homogeneity of thick disk, ULIRGs (ultra luminous infrared galaxies) do not evolve onto the Fundamental Plane, Scuba sources look like ellipticals at high redshift with very intense SFR, boxiness requires $\mathrm{E}-\mathrm{E}$ mergers.

Rosa Dominguez argued that in her simulations of galaxy formation in the $\Lambda$ CDM scenario ellipticals can form at early times and the massive ellipticals on shorter timescales than the smaller ellipticals. Silk argued that it is not correct to oppose the two scenarios and that the hierachical clustering scenario is essentially a model for the dark matter. Matthias Steinmetz said that quantitative calculations to fit the $[\alpha / \mathrm{Fe}]$ ratios with hierarchical models have never been performed because of the difficulty of obtaining a good numerical resolution, but that certainly galaxies did not form out of a collapsing sphere! (we all agreed on that!). However, he said that not necessarily HC models would predict the opposite of what is observed. In fact, he pointed out that although massive ellipticals would form later, this can be different in cluster environments where massive galaxies would form faster. As most of ellipticals are in clusters this could give rise to timescales in agreement with the observed $[\alpha / \mathrm{Fe}]$ versus velocity dispersion relation.Then he said that the spirals which merge to form the ellipticals are different from the present day spirals and that not all ellipticals form from spiral mergers. Cristina Chiappini then replied that if there 
is a difference in the formation timescales for ellipticals in clusters and in the field, then we should see different chemical properties and this is not observed as shown by Bernardi et al. (1998). Kobayashi said that in the $\Lambda \mathrm{CDM}$ scenario is still possible to have monolithic ellipticals. She also suggested that the presence of abundance gradients in ellipticals argue against the formation of ellipticals by mergers, whereas, on the contrary, ellipticals with no gradients can derive from mergers. Steven Shore recommended to include mergers in chemical evolution models before claiming that the real scenario cannot be hierarchical. He suggested in particular that chemical evolution models could test the infall timescales coming from simulations of galaxy formation. Cristina Chiappini replied that in the specific case of the Milky Way, the infall law suggested by Sommer-Larsen et al. (2002) in his HC model seems to be in good agreement with the infall law used in chemical evolution models which are able to reproduce the majority of the observational constraints. Hensler wondered whether dissipational mergers of spirals may create ellipticals in a way similar to monolithic collapse. However, Burkert said that his models for elliptical formation do need major mergers.

What emerged from this discussion was that in the future we need to combine detailed chemical evolution with semi-analytical models of galaxy formation in order to try to understand the many still open questions concerning galaxy formation and evolution.

In concluding, I would like to thank all of those who partecipated to the discussion and apologize if I missed other contributions to the discussion, since everybody has contributed to a very lively discussion.

\section{References}

Arimoto, N. \& Yoshiı, Y., 1987, A\&A 173, 23

Barbuy, Ortolani, S., Bica, E., 1998, A\&AS 132, 333

Barbuy, B., Grenon, M. 1990, in "Bulges of Galaxies", ESO/CTIO Workshop, eds. B.J. Jarvis and D.M. Terndrup, p. 83

Bender, R., Ziegler, B. \& Bruzual, G., 1996, ApJ 463, L51

Boissier, S., Boselli, A., Prantzos, N., Gavazzi, G., 2001, MNRAS 321, 733

Bernardi, M., Renzini, A., da Costa, L. N.., Wegner, G. \& Al., 1998, ApJ 508, L143

Blain, A. W., Smail, I., Ivison, R. J. \& Kneib, J.-P., 1999, MNRAS 302, 632

Boissier, S. \& Prantzos, N., 1999 MNRAS 307, 857

Bower, R. G., Lucey, J. R. \& Ellis, R. S., 1992, MNRAS 254, 601

Calura, F., Matteucci, F. \& Vladilo, G., 2002, MNRAS submitted

Chiappini, C., Matteucci, F. Gratton 1997, ApJ 477, 765

Chiappini, C., Matteucci, F. \& Padoan, P., 2000, ApJ , 528, 711

Chiappini, C., Matteucci, F., \& Romano, D., 2001, ApJ 554, 1044

Daddi, E., Cimatti, A., Renzini, A., 2000, A \&A 362, L45 
Ferreras, I. , Charlot, S. \& Silk, J., 1999, ApJ 521, 81

Franceschini, A., Silva, L., Fasano, G., Granato, L., Bressan, A., Arnouts, S., DANese, L., 1998, ApJ 506, 600 MNRAS 296, 709

Gonzalez, J.J., 1993, Ph.D. thesis. Univ. Calif., Santa Cruz

Kauffmann, G., Charlot, S. \& White, S. D. M., 1993, MNRAS 283, L117

Kauffmann, G., 1996, MNRAS 281, 475

Kodama, T., Arimoto, N., Barger, A. J. \& Aragon-Salamanca, A., 1998, A\&A 334, 99

Hill, V., François, P., Spite, M., Primas, F., Spite, F., 2000, A \&A 364, 19

LARSON, R.B., 1974, MNRAS 169, 229

Maraston, C., Thomas, D., 2000, ApJ 541, 126

Matteucci, F., 2001, The Chemical Evolution of the Galaxy, ASSL, Kluwer Academic Publisher

Matteucci, F., 1994, A\&A 288, 57

Matteucci, F., Molaro, P. \& Vladilo, G., 1997, A\&A 321, 45

Matteucci, F., Pipino, A., 2002, ApJ, 569, L69

Matteucci, F. \& Tornambè, A., 1987, A\&A 185, 51

McWilliam, A. \& Rich, R. M., 1994, ApJS 91, 749

Menanteau, F., Ellis, R.S., Abraham, R.G., Barger, A.J. \& Cowie, L.L., 1999, MNRAS 309, 208

Peebles, P.J.E. 2002, in "a New Era in Cosmology", ASP Conf. eds. N. Metcalf \& T. Shanks, astro-ph/0201015

Pettini, M., Rix, S.A., Steidel, C. C., Adelberger, K. L., Hunt, M. P., Shapley, A. E., 2002, ApJ 569, 742

Pipino, A., Matteucci, F., Borgani, S. \& Biviano, A., 2002, NewA 7, 227

Recchi, S., Matteucci, F. \& D'Ercole, A., 2001, MNRAS 322, 800

Renzini, A. \& Ciotti, L., 1993, 416, L49

Sommer-Larsen, J., Gotz, M., Portinari, L., 2002, astro-ph/ 0204366

Stanford, S. A., Eisenhardt, P. R. \& Dickinson, M., 1998, ApJ 492, 461

Steidel, C.C., Adelberger, K.L., Giavalisco, M., Dickinson, M., Pettini, M., 1999, ApJ 519, 1

Thomas, D., 1999, MNRAS 306, 655

Thomas, D., Maraston, C. \& Bender, R., 2002, Reviews in Modern Astronomy Vol. 15, Astronomische Gesellschaft, R.E. Schielicke (ed.), astro-ph/0202166

Trager, S. C., Faber, S. M., Worthey, G. \& GonzÀlez, J. J., 2000, AJ 120, 165

Trager, S. C., Worthey, G., Faber, S. M., Burstein, D. \& Gonz?lez, J. J., 1998, ApJS 116, 1

van Dokkum, P. G. \& Franx, M., 1996, MNRAS 281, 985

Vladilo, G., 2002, A \&A 391, 407

ZEPF, S.E., 1997, Nature 390, 377 